\title{
Review Article \\ Research on the Progress of Interdigital Transducer (IDT) for Structural Damage Monitoring
}

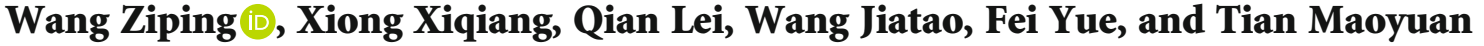 \\ Faculty of Civil Engineering and Mechanics, Jiangsu University, Research on Structural Health Management of Critical Components, \\ Zhenjiang, Jiangsu Province, China 212013 \\ Correspondence should be addressed to Wang Ziping; wzpxx2004@126.com
}

Received 3 October 2020; Revised 25 January 2021; Accepted 5 March 2021; Published 22 March 2021

Academic Editor: Giuseppe Maruccio

Copyright (C) 2021 Wang Ziping et al. This is an open access article distributed under the Creative Commons Attribution License, which permits unrestricted use, distribution, and reproduction in any medium, provided the original work is properly cited.

In the application of Structural Health Monitoring (SHM) methods and related technologies, the transducer used for electroacoustic conversion has gradually become a key component of SHM systems because of its unique function of transmitting structural safety information. By comparing and analyzing the health and safety of large-scale structures, the related theories and methods of Structural Health Monitoring (SHM) based on ultrasonic guided waves are studied. The key technologies and research status of the interdigital guided wave transducer arrays which used for structural damage detection are introduced. The application fields of interdigital transducers are summarized. The key technical and scientific problems solved by IDT for Structural Damage Monitoring (SHM) are presented. Finally, the development of IDT technology and this research project are summarised.

\section{Introduction}

The construction of important facilities in key engineering areas (such as transportation engineering, aerospace engineering, and civil engineering) is directly related to people's livelihoods and is the lifeblood of the national economy. These key facilities are inevitably affected by imperfect design theory, environmental load, fatigue, corrosion, and the aging of materials, which will inevitably result in damage accumulation and functional decline, which shortens the service life of the structures. It is even more important to detect and manage the health and safety of these large structures. For routine maintenance of engineering structures, the visual method is usually combined with other, conventional, nondestructive testing (NDT) methods such as radiography, ultrasound, or magnetic particles. Although the existing detection methods all have their own characteristics [1], it can meet the needs of damage detection in many engineering facilities. However, for existing, large-scale engineering facilities comprising structures made of composite/complex materials, the existing NDT methods cannot meet the needs of rapid and accurate extraction of minor damages from the structure. The latest research shows that new ultrasonic, guided-wave detection technology has the characteristics of long-distance transmission, sensitivity to small amounts of damage, controllable processes, etc. The industry has made considerable progress in the field of structural damage detection [2]. At present, there have been many studies on the damage detection of composite materials based on ultrasonic guided wave, which is also the focus of current studies [3-5]. However, how to apply it to engineering practice is still a problem Therefore, in view of the health and safety of large structures, the related theories and methods of Structural Health Monitoring (SHM) based on ultrasonic guided waves are studied and applied to the fields of vehicles, ships, aerospace structures, bridges, pipelines, and offshore platforms. In order to better understand the decline of structural function, real time informs users of tiny damage initiation and monitoring the remaining service life of structures ensures the safety and reliability of engineering structures in service, significantly reducing the maintenance cost of key structures and high-end equipment, which is still the main challenge for experts in the science and technology field. 
In recent years, IDT [6] has gradually been applied to SHM systems because of its characteristics, e.g., low frequency, low cost, adjustable frequency band, and low loss. The most common form is the formation of ultrasonic Lamb wave IDT, which can quickly detect a wide range. It has been applied in the damage detection of platy structures. Ultrasonic guided waves can propagate over a long distance with very small attenuation and are sensitive to various types of damage, which makes them suitable for the detection of large-area plate structures [7-9]. The selection of frequency and mode is of great significance for the detection of different damage types for composite plate structures [10-17]. Due to its advantages such as high sensitivity and wide range of detection, ultrasonic phased array has seen a sharp increase in its application in the field of NDT in recent years [18, 19]. The new flexible transducer array (with high electrical energy density, broadband, and omnidirectional excitation and reception) has been developed for large composite panel structure health assessment technology, so as to enhance its electrical coupling performance and achieve a wide range of exploration performance. This represents a further improvement of the existing SHM method. It will provide new sensor technology support for future smart materials and structures in aerospace and other large structural NDT applications, as well as promoting the application of new material devices and related technological developments.

\section{Research Progress of Ultrasonic Transducer Array for SHM}

Ultrasonic guided waves testing is a technique successfully used in many industrial scenarios worldwide [20]. As a nondestructive testing technology, ultrasonic phased array is widely used in the detection of cracks and other damages in engineering structures [21]. The existing SHM system, based on ultrasonic guided waves, often uses an embedded or sticker ultrasonic transducer unit to construct a sensor array with a certain spatial distribution or a sparsely distributed sensor network. It can be used to overcome the problem of impedance decay and low signal response in large area panels or composite structures [22]. The sparse distribution sensor network detects the local damage of the structure based on the transit time information of guided wave signals between sensor units; the accuracy of the damage imaging localisation greatly depends on the sparse sensor array [23-26]. In order to minimise the power consumption of the SHM system and the structural area occupied by the transducer, it is necessary to minimise the number and the occupied area of the transducer, while maintaining the ability of large-area scanning defects [27]. This can be solved by using linear or annular phased array transducers on structural surfaces or embedded structural surfaces. The authors [28] propose a low frequency ultrasonic phased array transducer composed of 16 OPCM (Orthotropic Piezoelectric Composite Material) components. By studying the total displacement variation of different parameters at the focal point of concrete structure, the optimal parameters such as the spacing, width, and number of elements of phased array are obtained. The ultrasonic phased array detection method is based on compact array transduc- ers, which can easily realise the deflection and focusing of stronger energy beams by controlling the delay of each unit signal. Linear scanning, sector scanning, and dynamic depth focus scanning easily detect defects in different directions and positions in a wide range of complex workpieces. The imaging results are intuitive [29]. Yu and Giurgiutiu [30] proposed the beam-forming model, to generate the propagation wave field formed by the omnibearing point wave source. Figure 1 shows a rectangular scan array and a circular scan array, which can realise the focused scan in the plate structure. Compared with the linear array [31] and the rectangular array, the polar array $[13,32]$ has better beam consistency in all directions and higher volumetric accuracy $[33,34]$.

The current use of piezoelectric ceramic transducers (PZT) to excite ultrasonic guided waves has the advantages of a high piezoelectric strain constant, fast response, and stable performance. However, inherent brittleness and the poor design of mechanical and electrical properties make it difficult to integrate or paste on the complex shape of the structural surface, which seriously affects the application scope of the PZT $[35,36]$. The baroclinic wedge transducer, comb transducer, and electromagnetic acoustic transducer (EMAT), which are used for guided wave excitation, can play a better role in off-line maintenance inspection. Due to its large scale, it is difficult to be integrated into the structure and can only be used for testing. In order to overcome the shortcomings of PZT's brittleness, it has better surface conformability in the curved shell structure [37]. Many scholars have paid attention to the application of flexible transducers in SHM systems [38]. Polyvinylidene fluoride film (PVDF) is a highly reactive thermoplastic fluoropolymer with strong piezoelectric effects, which can be used to make piezoelectric materials and electrostrictive materials. Also, it has the advantages of corrosion resistance and oxidation resistance, which was first discovered by Japanese scientist Kawai [39]. Compared with PZT, PVDF is more suitable for dynamic modal testing, which is flexible and easy to integrate; however, it cannot generate a large driving force due to its low elastic modulus when used for actuators [40]. For this purpose, Monkhouse et al. [41] designed a PVDF film with copper backing to improve the response characteristics of PVDF film and produce an interdigital electrode pattern using printed circuit board (PCB) technology; simulation defects are detected by modal selectivity. Badcock and Birt [42] added to the PZT powder in the epoxy resin substrate to form the polarized membrane, which is used to excite and receive guided waves. Performance is better than that of the same size PVDF film but it can still not achieve the simple PZT piezoelectric property. Therefore, when the transducer material is applied to the polar array, the power requirement will be very high. It is necessary to design an array structure that can be used to scan the whole of the surface area by 360 degrees, in the case of minimum power. However, the weak mechanical coupling caused by the weak drive capability and low rigidity of the PVDF film limits its application as a transducer material. Concerning the shortage of PVDF film, two kinds of flexible piezoelectric composites (AFC) and (MFC) have been developed. Bent and Hagood [43] were the first to propose that AFC devices are mainly used for 


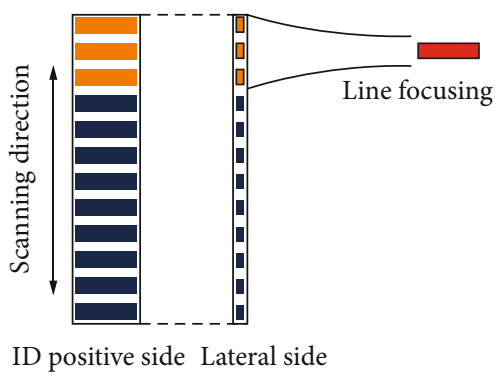

(a) Linear array

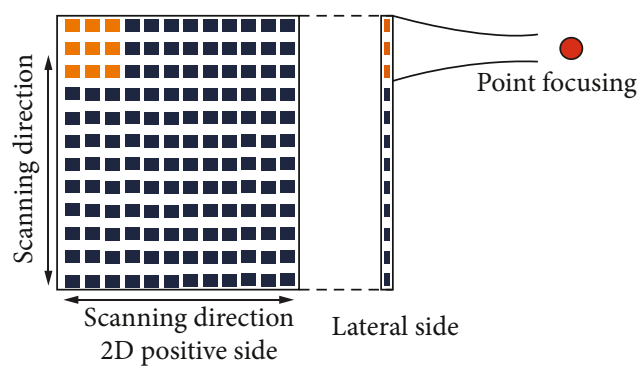

(b) Rectangular array

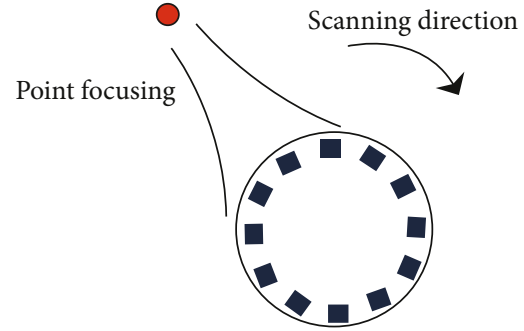

(c) Polar array

Figure 1: Phased array transducer.

the active drive of structure and can also be used for sensing. From the NASA Langley Research Center, we have studied the same MFC device shown in Figure 2(a), which is an active driving element, consisting of unidirectional fibers and polymer matrixes. However, the fiber passing through is a square [44]. The interdigitated structure (IDT) mode of the transducer is matched with a certain wavelength. The polymer layer makes the flexibility of the transducer increase and the stress wave with high directivity is generated along the length direction of the piezoelectric fiber on the piezoelectric substrate (which shows obvious anisotropy) and its driving ability is greatly enhanced in a certain direction.

In 2018, Dame [45] studied the surface acoustic wave interdigital transducer (SAW-IDT), which has great potential for the characterisation of thin layers, coatings, and functional surfaces. In order to optimise SAW-IDT, it is necessary to study various SAW-IDT configurations by changing the number, size, shape, and spacing of electrodes. Dame [46] (and others) have developed and optimised the excitation of the Surface Acoustic Wave (SAW) broadband fork, referred to in the transducer (IDT), in order to cover the large bandwidth between 20 and $125 \mathrm{MHz}$. The spectral distribution of SAW was adjusted by optimizing the spatial distribution (width and spacing) of the electrode and an IDT transducer can be used to measure the displacement of surface sound waves, propagating over a distance of more than $20 \mathrm{~mm}$. In 2018, Zhong [47] chose blackened, reduced lithium tantalate wafers as the substrate material and made use of the stripping process, to prepare the interdigital transducer (IDT). The electrical performance test and reliability verification of the $2,492 \mathrm{MHz}$ acoustic meter filter were carried out. Lavish [48] used surface acoustic wave (SAW) to detect microdamage through an anisotropic piezoelectric sensor. A novel technique based on Rayleigh wave single input and multiple output is proposed to detect microcracks/defects in sensors, and a damage detection algorithm, based on empirical mode decomposition (EMD) and principal component analysis (PCA), should be implemented to quantify damage evolution in piezoelectric sensor materials. In 2019, our team [49] proposed the study of a trapezoidal interdigital transducer (TIDT) for the lack of bandwidth in existing rectangular IDT. The structure of TIDT is optimised by finite element analysis, and the performance of the designed TIDT is tested. The preliminary application of TIDT to structural damage detection is studied. Compared with the insufficient brittleness of PZT material, the flexibility of PVDF is more suitable for dynamic modal testing and is easy to integrate. The results show that the sensing performance of TIDT based on PVDF is better than that of PZT-5A. Figure 3 shows the displacement in $x$ direction with TIDT based on PVDF. The smaller the electrode spacing, the greater the displacement response. The displacement in $x$ direction of PVDF base is greater and it has broadband characteristics. The PVDF thin film is limited by its low piezoelectric constant and poor driving performance. Therefore, it is urgent that we find a new, flexible material as an IDT substrate for better driving performance. In 2020, Chen [50] proposed the preparation of IDT using graphene. The sensitivity enhancement and quadratic effect of the sensor were studied by the finite element method (FEM). It was proved that graphene IDT had superior sensing performance.

Dielectric elastomer (DE) is a new type of flexible, electro-induced, smart polymer material with high electromechanical conversion efficiency; it can overcome the weak driving force of PVDF and can be used for excitation and reception functions. DE has the advantages of flexibility, easy forming, and fatigue damage. Since the 1990s, many scholars have carried out experimental research on DE materials from performance to model [51-55], as well as in the aerospace, medical, health, and robotics sectors. Dielectric elastomer transducers are a class of electroactive polymers that operate based on the interaction of quasistatic electric charges with deformable dielectric and electrode materials [56]. In 1998, SRI first attempted to apply DE materials to design actuators and found that DE actuators had the best comprehensive performance compared to other types of drives [57]. As shown in Figure 2(b), the simplest application of DE transducers is that they are coated with flexible electrodes on both sides of thin, compliant DE, which can be used for actuators and strain sensors. Different DE materials directly affect their actuating and sensing performance. For ultrasonic guided wave detection, environmental conditions such as temperature have an important impact on ultrasonic Lamb wave damage detection, thus bringing more possibilities to the design of transducer of $\mathrm{DE}$ material [58]. At present, the most commonly used are acrylic ester materials that can obtain higher energy density. Neukasil RTV-23, DC3481 (and other types of silicone rubber (PDMS) and polyurethane (PU)) are also kinds of DE material that adapts to wide temperature variation, high conversion effect, and fast 


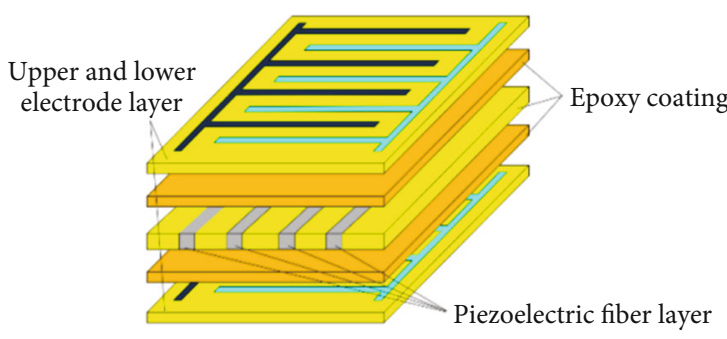

(a) MFC structure transducer

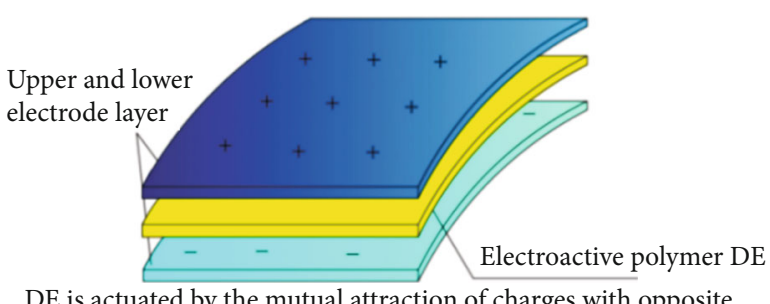

$\mathrm{DE}$ is actuated by the mutual attraction of charges with opposite

(b) DE structure transducer

FIgURE 2: Schematic diagram of MFC and DE structure.

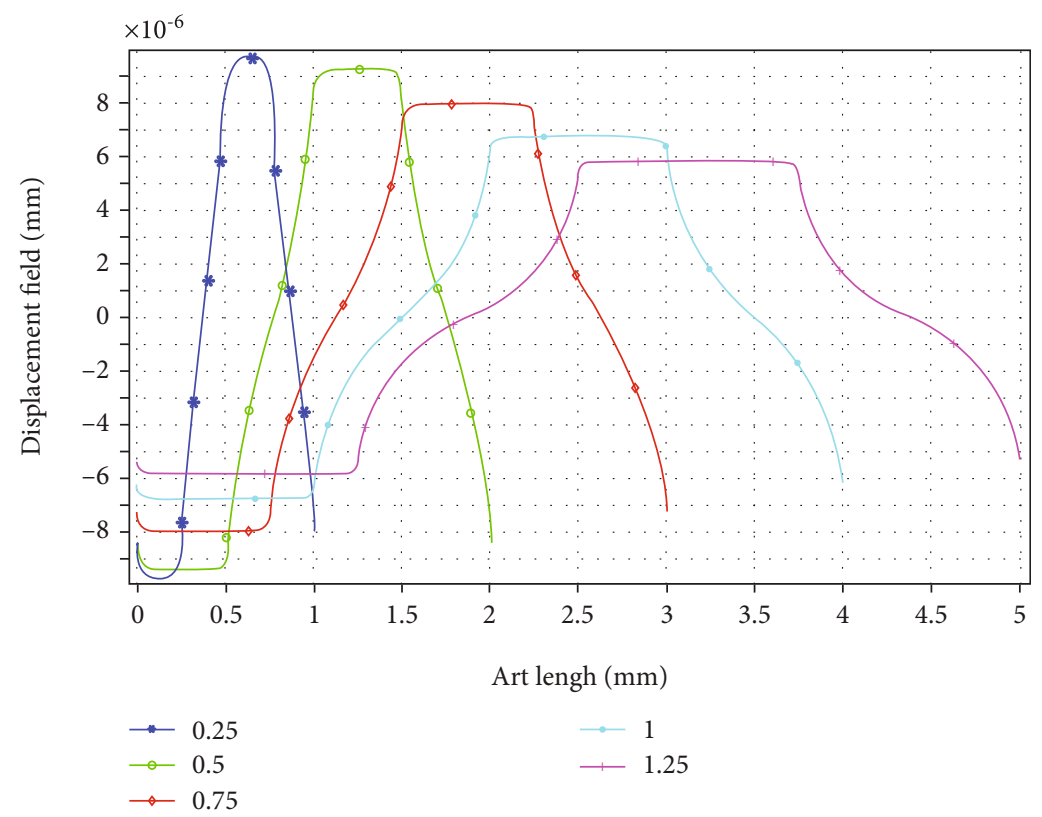

Figure 3: The displacement in $x$ direction with TIDT based on PVDF.

response [59]. In 2014, Araromi et al. [60] formed a polydimethylsiloxane carbon electrode by laser ablation and, then, achieved a permanent and firm combination with the silicone rubber film through oxygen plasma activation. The electrode has high resolution and high robustness, which can be used in DE actuators and sensors. In 2015, Oluwaseun et al. developed a flexible, interdigital, capacitive DE sensor using a new preparation process of polydimethyl siloxanes (PDMS), to form a carbon composite electrode. In 2018, Philip et al. [61] studied the reasonable optimisation of DE and compliant electrode materials when the dielectric constant and conductivity changed. They concluded that the $\left[\mathrm{R}_{\mathrm{n}} \mathrm{SiO}_{4-\mathrm{n} / 2}\right]$ elastomer has low-leakage current density, extremely low glass transition temperature, and very fast electromechanical response; it can be considered as a future replacement for polydimethyl siloxanes elastomer in DE sensors. In 2019, our team [62] proposed an omnidirectional, broadband, flexible, dielectric interdigital transducer, and its manufacturing method. By changing the distance between compliant, annular, interdigital electrodes, the IDT wideband characteristics are realised, making the frequency bandwidth designable, thereby achieving guided wave mode selection and improving the recognition rate of effective sig- nals in damage detection in engineering structures. Thus, the tested omnidirectional driving component and sensing of midultrasonic signals was achieved. Therefore, through different DE and compliant electrode materials, a flexible actuating/sensing device with different application environments has been developed $[63,64]$. The team has developed a new type of actuator for SHM using propylene acrylate DE material (VHB4910) instead of traditional piezoelectric materials, which has realised a high voltage driving and high sensitivity sensing function. A transducer unit can be used to achieve wide range detection.

\section{Research Progress of IDT Technology}

IDT technology is derived from the development of surface acoustic wave (SAW) devices. In recent years, it has been gradually transferred to guided-wave detection, based on the SHM system. The IDTs shown in Figure 4(a) have the characteristics of comb electrodes, which can be placed either on one side or both sides of the piezoelectric substrate, because the frequency characteristics of IDT correspond to the geometric structure of the IDT fingers. IDT relies on a pair of comb electrodes to have the function of modal 


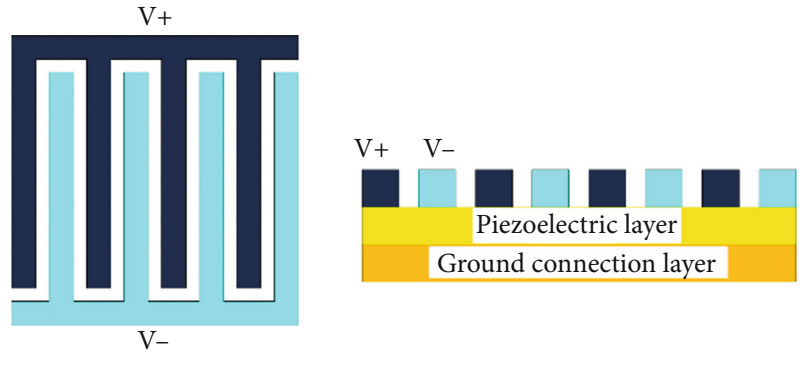

(a) Conventional single-sided electrode

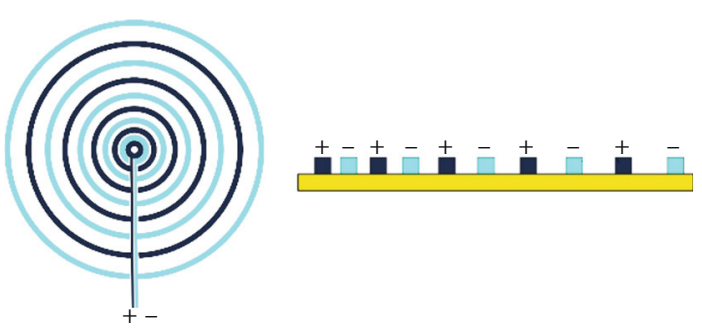

(b) Annular variable pitch single sided electrode

FIgure 4: IDT transducer.

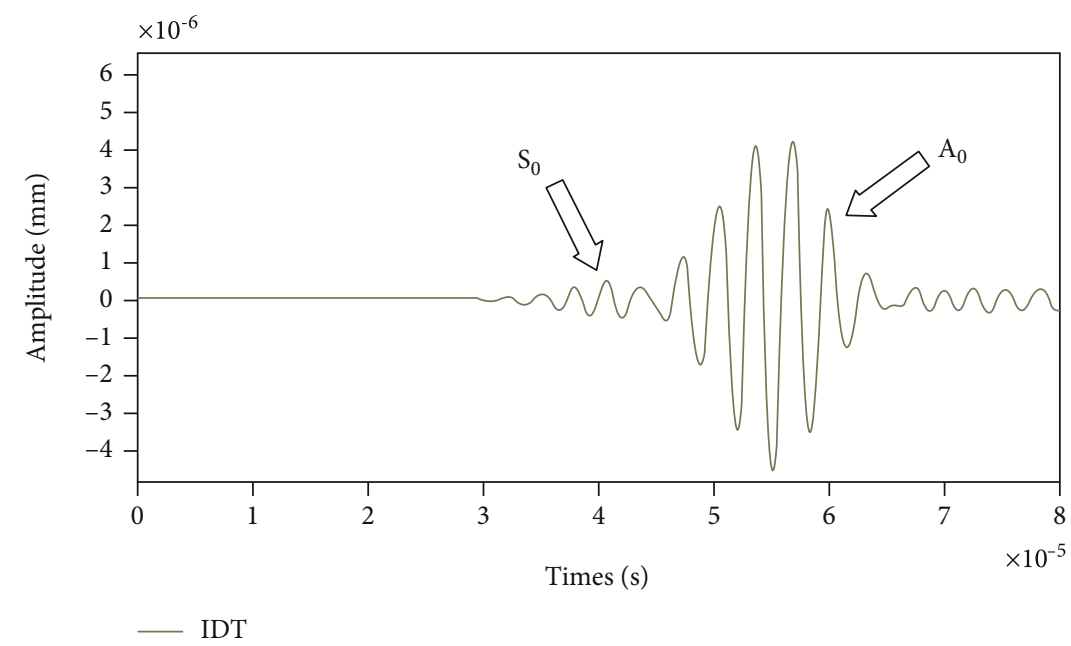

FIGURE 5: Guided wave mode selection [35].

selection [65-67]. Figure 4(b) is based on the design of the Chirp interdigital transducer [68] with a wide frequency band as an annular variable pitch and a single-sided IDT with wideband and mode selection functions.

Because the IDT transducer can form a directional driving function through the arrangement of interdigital electrodes, the advantage of low sidelobe level guided waves can be generated based on the structure, which does not require complex electrode driving. Quek et al. [69] constructed an IDT transducer that generates guided waves for NDE and verified that the IDT transducer's detection and recognition ability is superior to ordinary piezoelectric transducers, and has been successfully used in flat and curved structures. Wilcox et al. [70] tried to successfully construct an IDT on the PVDF membrane substrate for guided waves in order to transmit and receive from pipelines and investigated their ability to apply phase velocity and group velocity attenuation curves for guided wave mode selection. The finite element method (FEM) verified the drive capability of the $A_{0}$ modal guided wave preset at $0.95 \mathrm{MHz}$ shown in Figure 5. The interdigital transducer (IDT) designed by Hayward et al. [71] is a piezoelectric composite layer between two PCBs and can also achieve the choice of wave number and mode shape. Based on the theory of a wavelength-adjustable section actuator, the flexibility of a guided wave test operation point can be guaranteed. Wilcox [72] proposed the concept of a circular array of 6 PVDF flexural interdigital IDT, so that each element will generate divergent sound beams, which can check the sector slice area and the detection range of 360 degrees.

However, the directivity of IDT transducers depends on the exponent of the interdigital electrodes, the excitation frequency, and the piezoelectric constant $d_{31}$ or $d_{33}$ of the composites. Currently, commercially available flexible MFC is produced by the American Intelligent Materials Company. In 2000, a project group began to study the 1-3 type piezoelectric fiber composite components and has gradually applied the results of independent intellectual property rights to structural damage detection [73-75]. In recent years, the most commonly used IDT transducers have been developed on two structures, AFC and MFC, in the form of equal distance IDT transducers [76], weighted IDT transducers [77], trapezoidal IDT transducers [78], circular IDT focusing transducers, and helix-shaped, directional transducers. Table 1 shows the advantages and disadvantages of each. In many different forms of IDT topology design, the circular and spiral IDT transducers can be used to solve the omnidirectional and frequency selection problems in Lamb wave detection. It is suitable for flexible arrays. A hot topic in the research of guided wave excitation is the fact that a ring IDT focusing transducer can produce an ultrasonic guided wave with a high intensity and high beam width compression ratio, which is very suitable as a source of microacoustic 
TABLE 1: Advantages and disadvantages of IDT.

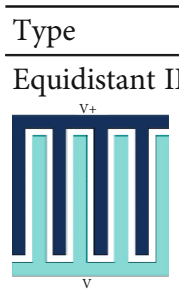

Weighted IDT [77]

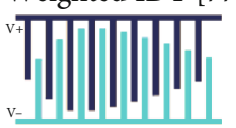

Trapezoidal IDT

Transducer [78]

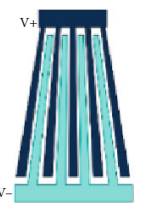

Polar focusing IDT [81]

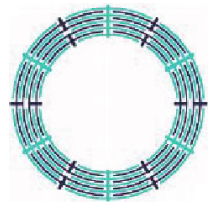

\section{Ultrasonic motor, optical scanning,} and damage detection

Filter and design different excitation response
High sensitivity and directivity

$D_{33}$ and $D_{31}$ mode can be adopted.

Design different excitation response

Broadband and high sensitivity

Omnidirectional detection, damage detection, and modal selection

The excitation frequency is selected to Large area, multi damage, sensor, and realise continuous sound beam steering surface structure can be detected. and directional excitation, and sound beam intensity compensation.
Insufficient

Single frequency

Inconsistent performance

Strip width and loss

Single direction of encouragement

Helical MFC directional transducer [79]

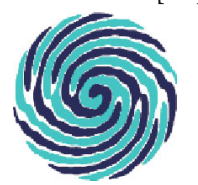

Omnidirectional

circular broadband IDT

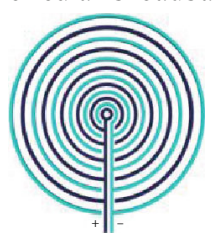

Detection of large area curved metal sheets and composites $360^{\circ}$ omnidirectional transmitting and receiving. Frequency bandwidth is designed so that it has modal selectivity.
The design is complex, different materials need to be changed, and the sensitivity is low.
The signal response depends on the length of the interdigital, the low actuating voltage, and the nonphased array detection.

Cannot be used for directional scanning and receiving. channel or waveguide. Their basic characteristics are only controlled by their geometry. It is necessary to analyse their diverse geometry [79]. However, there are still many shortcomings in IDT design, such as narrow bandwidth and single directivity. In order to adapt to a smaller scale of damage detection, smart transducer structural design is needed to enhance the bandwidth to realise the omnidirectional actuating and sensing [80].

$\mathrm{DE}$ is an electroactive polymer with great strain capacity. The basic functional components of DE transducers are thin films sandwiched between electrodes. DE and compliant materials are used to replace conventional PZT and silver paste electrode materials. Through reasonable material selection and design, the actuating and sensing sensitivity of transducers can be greatly improved. The basic principle is shown in Figure 6. Like conventional piezoelectric materials, the performance of the DE transducer depends on the mechanical and electrical boundary conditions. The same charge Q, in Figures 7(a) and 7(b), is used as the basic equation for the transducer, as follows:

$$
\begin{gathered}
C=\varepsilon_{0} \varepsilon A / z, \\
E_{e}=0.5 Q^{2} / C, \\
P_{e f f}=\frac{\varepsilon_{0} \varepsilon V^{2}}{z^{2}} .
\end{gathered}
$$

In the formula, $C$ is electric capacity, $E e$ is electrical energy, $P_{\text {eff }}$ is effective pressure, $\varepsilon_{0}$ is the absolute permittivity 


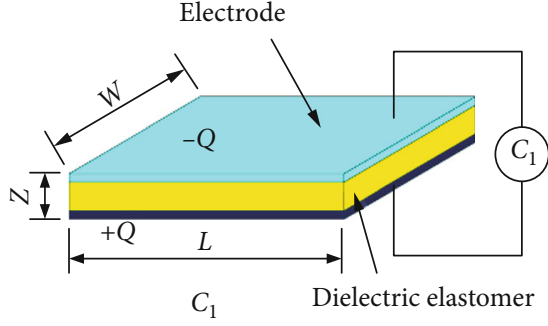

(a) After deformation

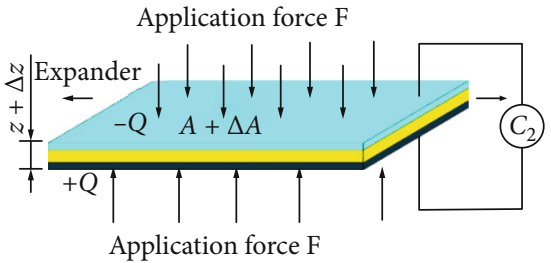

(b) After deformation

FIGURE 6: Working principle of DE transducer.

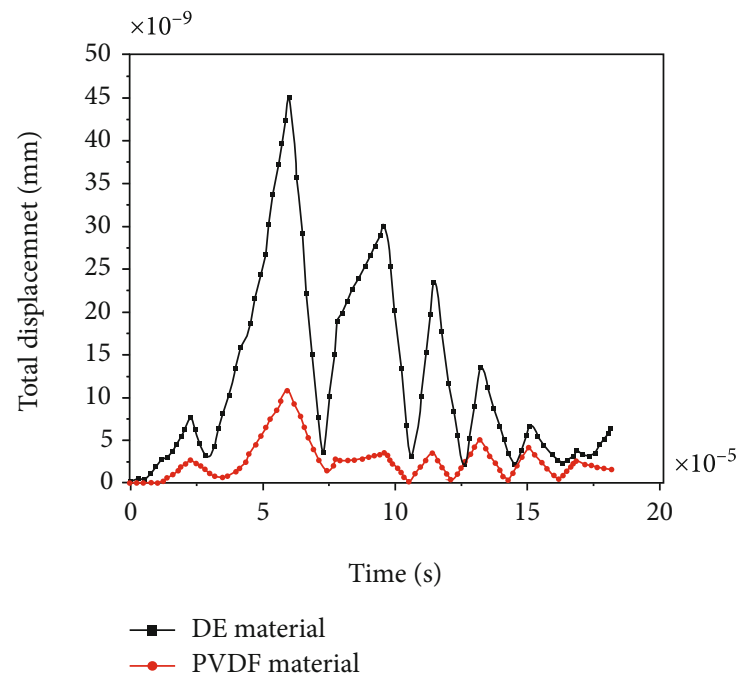

Figure 7: $z$ direction displacement components received by transducers driven by different substrate materials at the same point.

of vacuum $\left(\varepsilon_{0}=8.85 * 10^{-12} \mathrm{~F} / \mathrm{m}\right), A$ is the area sandwiched between electrodes, and $z$ is the thickness direction of the thin film. The electric energy and voltage will change, along with the stretching or shrinking of the material. From equation (3) $V=Q / C$, it is known that when the thickness changes, it will convert electric energy to mechanical energy at a given rate. When the elastic restoring force balances effective pressure $P_{\text {eff }}$, the material is in equilibrium. If $P_{\text {eff }}$ is greater than the elastic restoring force, DE will brake through the contraction thickness $Z$, and convert electrical energy into mechanical energy. On the other hand, if $P_{\text {eff }}$ is smaller than elastic resilience, the elastomer will expand the thickness and convert mechanical energy into electrical energy.

Research on dielectric elastomers as a DE-IDT substrate has been carried out. In the case of the same excitation and the same arrangement of the interdigital electrode, the fivepeak wave with the frequency $450 \mathrm{kHz}$ was modulated by the Hanning window. In order to test the displacement response performance of two different substrate materials, DE and PVDF sheets with electrodes were, respectively, pasted on the aluminum plate at a displacement of $80 \mathrm{~mm}$ from the PZT sheet. Figure 7 shows the displacement response values used by DE and PVDF materials when PZT are used to act as an actuator. The $z$ direction displacement component received at the same point (with a dielectric elastomer as the base transducer) is 3 orders of magnitude higher than the PVDF base transducer. The actuating performance was greatly improved.

\section{IDT Technology Conclusion and Prospect}

Selecting the proper mode in a guided wave test is very important for scanning, separating, and characterising various structural defects. IDT can improve the defect of the original single mode imaging method, thereby improving the detection ability of the structural damage and constructing a corresponding experimental platform. The demand for theoretical development is also of great practical significance. Based on the structural, nondestructive testing of the key technology of an interdigital guided wave transducer array, we have studied the health and safety of large-scale structures and analysed the advanced theories and methods of advanced SHM based on ultrasonic guided waves, as well as studying the ultrasonic transducer arrays and interdigital transducers used in SHM.

(1) A new flexible transducer array with high power density, wideband and omnidirectional excitation and reception is developed, which can be applied to large, composite panel structure health assessment technology. It can improve its electromechanical coupling performance to achieve a wide range of exploration performance and further improve the existing SHM method

(2) Instead of traditional piezoelectric materials, propylene ester DE material (VHB4910) is used to develop a new actuator for SHM. It realises a high-voltage driving and high-sensitivity sensing function. A transducer unit can be used to realise wide range detection

(3) Replace conventional PZT and silver slurry electrode materials with $\mathrm{DE}$ and supple electrodes and use them for IDT development. The material is used in the development of IDT and multidevice arrays. The actuating and sensing sensitivity of transducers can be greatly improved by rational material selection and design. The integration of transducers into materials and intelligentisation will hopefully promote the development and wide application of micronanosensors and energy harvesters 


\section{Data Availability}

The processed data required to reproduce these findings cannot be shared at this time as the data also forms part of an ongoing study.

\section{Conflicts of Interest}

The authors declare that they have no conflicts of interest.

\section{Acknowledgments}

This work was financially supported by the National Natural Science Foundation of China (11872191 and 11520101001) and the Postgraduate Research \& Practice Innovation Program of Jiangsu Province (KYCX20_3073).

\section{References}

[1] S. Takeshi, A. Mitunori, and F. Shinichi, "Develpoment of non-destructive inspection technology of titanium," Nippon Steel Technical Report, vol. 85, pp. 157-161, 2002.

[2] X. Y. Wang, Y. Y. Yang, and D. S. Wang, "Design of ultrasonic guided wave excitation signal generator for rotating shafts," Advanced Materials Research, vol. 629, pp. 570-575, 2012.

[3] D. Le, J. Lee, Y. Cho, D. K. Dao, T. G. Nguyen, and H. Phan, "Ultrasonic guided waves in unidirectional fiber-reinforced composite plates," in Advances in Condition Monitoring and Structural Health Monitoring, pp. 677-685, Springer, 2021.

[4] K. A. Tiwari, R. Raisutis, O. Tumsys, A. Ostreika, K. Jankauskas, and J. Jakutavicius, "Defect estimation in nondestructive testing of composites by ultrasonic guided waves and image processing," Electronics, vol. 8, no. 3, p. 315, 2019.

[5] S. Sikdar and S. Banerjee, "Identification of disbond and high density core region in a honeycomb composite sandwich structure using ultrasonic guided waves," Composite Structures, vol. 152, pp. 568-578, 2016.

[6] X. Sun, W. Liu, X. Shao, S. Zhou, W. Wang, and D. Lin, "Surface acoustic wave gyroscopic effect in an interdigital transducer," Sensors, vol. 19, no. 1, p. 106, 2019.

[7] A. Raghavan and C. E. S. Cesnik, "Review of guided-wave structural health monitoring," The Shock and Vibration Digest, vol. 39, no. 2, pp. 91-114, 2007.

[8] Z. Su, L. Ye, and Y. Lu, "Guided Lamb waves for identification of damage in composite structures: a review," Journal of Sound and Vibration, vol. 295, no. 3-5, pp. 753-780, 2006.

[9] J. L. Rose, "Recent advances in guided wave NDE," in 1995 IEEE Ultrasonics Symposium. Proceedings. An International Symposium, pp. 761-770, Seattle, WA, USA, 1995.

[10] J. L. Rose, A. Pilarski, and J. J. Ditri, “An approach to guided wave mode selection for inspection of laminated plate," Journal of Reinforced Plastics and Composites, vol. 12, no. 5, pp. 536-544, 1993.

[11] T. Kundu, C. Potel, and J. F. de Belleval, "Importance of the near Lamb mode imaging of multilayered composite plates," Ultrasonics, vol. 39, no. 4, pp. 283-290, 2001.

[12] N. Guo and P. Cawley, "The interaction of lamb waves with delaminations in composite laminates," The Journal of the Acoustical Society of America, vol. 94, no. 4, pp. 2240-2246, 1993.
[13] D. N. Alleyne and P. Cawley, "The interaction of lamb waves with defects," IEEE Transactions on Ultrasonics, Ferroelectrics and Frequency Control, vol. 39, no. 3, pp. 381-397, 1992.

[14] K. Maslov and T. Kundu, "Selection of Lamb modes for detecting internal defects in composite laminates," Ultrasonics, vol. 35, no. 2, pp. 141-150, 1997.

[15] J. E. Michaels, S. J. Lee, J. S. Hall, and T. E. Michaels, "Multimode and multi-frequency guided wave imaging via chirp excitations," Proceedings of SPIE, vol. 7984, no. 6, p. 15, 2011.

[16] H. Cho, S. Choi, and C. J. Lissenden, "Effect of skew angle on second harmonic guided wave measurement in composite plates," in 43rd Review of Progress in Quantitative Nondestructive Evaluation, Atlanta, USA, 2016.

[17] H. Cunfu, L. Yan, S. Guorong, Y.-C. Lee, and W. Bin, "Multimode measurement of lamb wave propagating on thin isotropic metal sheet by line-focus PVDF transducer," in 2013 IEEE 11th International Conference on Electronic Measurement \& Instruments, pp. 542-546, Harbin, China, 2014.

[18] B. Shan, Z. Duan, and O. Jinping, "Study of ultrasonic phased array inspection imaging technology for NDT," China Welding, vol. 3, pp. 1-5, 2006.

[19] J. H. Kurz, A. Jüngert, S. Dugan, G. Dobmann, and C. Boller, "Reliability considerations of NDT by probability of detection (POD) determination using ultrasound phased array," Engineering Failure Analysis, vol. 35, pp. 609-617, 2013.

[20] S. Malo, S. Fateri, M. Livadas, C. Mares, and T. H. Gan, "Wave mode discrimination of coded ultrasonic guided waves using two-dimensional compressed pulse analysis," IEEE Transactions on Ultrasonics, Ferroelectrics, and Frequency Control, vol. 64, no. 7, pp. 1092-1101, 2017.

[21] L. Feng and X. Qian, "Enhanced sizing for surface cracks in welded tubular joints using ultrasonic phased array and image processing," NDT \& E International, vol. 116, p. 102334, 2020.

[22] V. Samaitis and L. Mažeika, "Influence of the spatial dimensions of ultrasonic transducers on the frequency spectrum of guided waves," Sensors, vol. 17, no. 8, p. 1825, 2017.

[23] B. Sorazu, G. Thursby, and B. Culshaw, "Wavefront integrating fiber sensors for ultrasonic detection," IEEE Sensors Journal, vol. 11, no. 7, pp. 1623-1631, 2011.

[24] J. E. Michaels and T. E. Michaels, "Guided wave signal processing and image fusion for in situ damage localization in plates," Wave Motion, vol. 44, no. 6, pp. 482-492, 2007.

[25] Z. Su, L. Cheng, X. Wang, L. Yu, and C. Zhou, "Predicting delamination of composite laminates using an imaging approach," Smart Materials \& Structures, vol. 18, no. 7, article 074002, 2009.

[26] J. E. Michaels, "Detection, localization and characterization of damage in plates with anin situarray of spatially distributed ultrasonic sensors," Smart Materials and Structures, vol. 17, no. 3, p. 035035, 2008.

[27] X. Ming, "Structure of a cylindrical high-power ultrasonic transducer," Acoustics and Electronic Engineering, vol. 92, no. 4, pp. 18-26, 2008.

[28] Z. Wang, Y. Luo, G. Zhao, B. Q. Xu, and F.-G. Yuan, "Design of an orthotropic piezoelectric composite material phased array transducer for damage detection in a concrete structure," Research in Nondestructive Evaluation, vol. 27, no. 4, pp. 204215, 2016.

[29] X. Chen, J. E. Michaels, and T. E. Michaels, "Design of distributed sparse arrays for Lamb wave SHM based upon estimated 
scattering matrices," AIP Conference Proceedings, vol. 1581, no. 1, pp. 248-255, 2014.

[30] L. Yu and V. Giurgiutiu, "In situ 2-D piezoelectric wafer active sensors arrays for guided wave damage detection," Ultrasonics, vol. 48, no. 2, pp. 117-134, 2008.

[31] V. Giurgiutiu and J. Bao, "Embedded-ultrasonics structural radar for in situ structural health monitoring of thin-wall structures," Structural Health Monitoring, vol. 3, no. 2, pp. 121-140, 2004.

[32] F. Yan and J. L. Rose, "Guided wave phased array beam steering in composite plates - art. no. 65320G," Proceedings of SPIE The International Society for Optical Engineering, vol. 6532, 2007.

[33] J. He and F. G. Yuan, "A quantitative damage imaging technique based on enhanced CCRTM for composite plates using 2D scan," Smart Materials and Structures, vol. 25, no. 10, p. 105022, 2016.

[34] J. He and F. G. Yuan, "Lamb wave-based subwavelength damage imaging using the DORT-MUSIC technique in metallic plates," Structural Health Monitoring: An International Journal, vol. 15, no. 1, pp. 65-80, 2016.

[35] F. Ciampa, S. G. Pickering, G. Scarselli, and M. Meo, "Nonlinear imaging of damage in composite structures using sparse ultrasonic sensor arrays," Structural Control \& Health Monitoring, vol. 24, no. 5, 2017.

[36] H. Y. Chang and F. G. Yuan, "Damage imaging in a stiffened curved composite sandwich panel with wavenumber index via Riesz transform," Structural Health Monitoring, vol. 19, no. 3, pp. 902-916, 2020.

[37] R. Joseph, B. Cody, O. Steve, and R. Alex, "Rapid large area inspection from a single sensor position: a guided shear wave phased array scan," Material Evaluation, vol. 75, no. 6, pp. 121-140, 2017.

[38] S. Yilong, L. Xiujuan, Z. Ye, Z. Hourong, and D. Zhang, "Preparation and characterization of lead zirconate titanate piezoelectric fiber and its composites," Science and Engineering of Powder metallurgy Materials, vol. 5, 2013.

[39] H. Kawai, "The piezoelectricity of poly (vinylidene fluoride)," Japanese Journal of Applied Physics, vol. 8, no. 7, pp. 975-976, 1969.

[40] A. Pal, A. Sasmal, B. Manoj, D. S. D. P. Rao, A. K. Haldar, and S. Sen, "Enhancement in energy storage and piezoelectric performance of three phase (PZT/MWCNT/PVDF) composite," Materials Chemistry and Physics, vol. 244, p. 122639, 2020.

[41] R. S. C. Monkhouse, P. D. Wilcox, and P. Cawley, "Flexible interdigital PVDF transducers for the generation of Lamb waves in structures," Ultrasonics, vol. 35, no. 7, pp. 489-498, 1997.

[42] R. A. Badcock and E. A. Birt, "The use of 0-3 piezocomposite embedded Lamb wave sensors for detection of damage in advanced fibre composites," Smart Materials and Structures, vol. 9, no. 3, pp. 291-297, 2000.

[43] A. A. Bent and N. W. Hagood, "Piezoelectric fiber composites with interdigitated electrodes," Journal of Intelligent Material Systems and Structures, vol. 8, no. 11, pp. 903-919, 1997.

[44] K.-C. T. Nguyen, L. H. Le, T. N. H. T. Tran, M. D. Sacchi, and E. H. M. Lou, "Excitation of ultrasonic Lamb waves using a phased array system with two array probes: phantom and in vitro bone studies," Ultrasonics, vol. 54, no. 5, pp. 11781185, 2014.
[45] D. Fall, M. Duquennoy, M. Ouaftouh, B. Piwakowski, and F. Jenot, "Effective and rapid technique for temporal response modeling of surface acoustic wave interdigital transducers," Ultrasonics, vol. 82, pp. 371-378, 2018.

[46] D. Fall, M. Duquennoy, M. Ouaftouh, N. Smagin, B. Piwakowski, and F. Jenot, "Optimization of interdigital transducers for the generation of surface acoustic waves over a large bandwidth (20-125 MHz)," Sensors and Actuators A: Physical, vol. 273, pp. 303-310, 2018.

[47] L. Zhong, Development of Beidou navigation $2492 \mathrm{MHz}$ low loss SAW filter, Southeast University, 2018.

[48] L. Pamwani, A. Habib, F. Melandsø, B. S. Ahluwalia, and A. Shelke, "Single-input and multiple-output surface acoustic wave sensing for damage quantification in piezoelectric sensors," Sensors, vol. 18, no. 7, p. 2017, 2018.

[49] C. Liangbin, Broadband TIDT structure optimization design for structural damage detection, Jiangsu University, 2019.

[50] C. Chen and J. Jin, "Surface acoustic wave vapor sensor with graphene interdigital transducer for TNT detection," Sensing and Imaging, vol. 21, no. 215, pp. 427-438, 2020.

[51] M. Mańka, A. Martowicz, M. Rosiek, T. Stepinski, and T. Uhl, "Tunable interdigital transducers made of piezoelectric macrofiber composite," JSmart Materials and Structures, vol. 25, no. 11, Article ID 115022, 2016.

[52] H. S. Kwon and J. Y. Kim, "An analytical filter design method for guided wave phased arrays," Mechanical Systems \& Signal Processing, vol. 81, pp. 433-446, 2016.

[53] F. Carpi, I. Anderson, S. Bauer et al., "Standards for dielectric elastomer transducers," Smart Materials \& Structures, vol. 24, no. 10, article 105025, 2015.

[54] B. Osmani, S. Seifi, H. S. Park, V. Leung, T. Töpper, and B. Müller, "Nanomechanical probing of thin-film dielectric elastomer transducers," Applied Physics Letters, vol. 111, no. 9, article 093104, 2017.

[55] B. Osmani, E. A. Aeby, and B. Müller, "Stress measurements of planar dielectric elastomer actuators," Review of Scientific Instruments, vol. 87, no. 5, Article ID 053901, 2016.

[56] R. Pelrine and R. Kornbluh, "Dielectric elastomers as electroactive polymers (EAPs): fundamentals," in Electromechanically Active Polymers, vol. 1, p. 17, Springer, 2016.

[57] R. E. Pelrine, R. D. Kornbluh, and J. P. Joseph, "Electrostriction of polymer dielectrics with compliant electrodes as a means of actuation," Sensors and Actuators A: Physical, vol. 64, no. 1, pp. 77-85, 1998.

[58] R. Gorgin, Y. Luo, and Z. Wu, "Environmental and operational conditions effects on Lamb wave based structural health monitoring systems: a review," Ultrasonics, vol. 105, p. 106114, 2020.

[59] F. B. Madsen, A. E. Daugaard, S. Hvilsted, and A. L. Skov, "The current state of silicone-based dielectric elastomer transducers," Macromolecular Rapid Communications, vol. 37, no. 5, pp. 378-413, 2016.

[60] O. A. Araromi, S. Rosset, and H. R. Shea, "Versatile fabrication of PDMS-carbon electrodes for siliconedielectric elastomer transducers," in 2015 Transducers - 2015 18th International Conference on Solid-State Sensors, Actuators and Microsystems (TRANSDUCERS), pp. 1905-1908, Anchorage, AK, USA, 2015.

[61] P. Caspari, S. J. Dünki, F. A. Nüesch, and D. M. Opris, “Dielectric elastomer actuators with increased dielectric permittivity and low leakage current capable of suppressing electromechanical instability," Journal of Materials Chemistry C, vol. 6, no. 8, pp. 2043-2053, 2018. 
[62] Z. Wang, Y. Luo, C. Xu, F. Yuan, B. Xu, and Z. Chiang, “An omnidirectional broadband flexible interdigital elastic transducer and its manufacturing method," China Patent: cn 109714018a, 2019.

[63] S. Sang, A. Mhannawee, and Z. Wang, "A design of active elastic metamaterials with negative mass density and tunable bulk modulus," Acta Mechanica, vol. 230, no. 3, pp. 1003-1008, 2019.

[64] S. Sang, A. Mhannawee, and Z. Wang, "Wave attenuation and negative refraction of elastic waves in a single-phase elastic metamaterial," Acta Mechanica, vol. 229, no. 6, pp. 25612569, 2018.

[65] F. Ge, D. Yang, M. Tian, N. Ning, W. Wu, and L. Zhang, "Research progress in the transformation of mechanical energy into electrical energy dielectric elastomer," Materials Science and Technology, vol. 24, no. 5, pp. 1-8, 2016.

[66] O. A. Araromi, S. Rosset, and H. R. Shea, "Versatile fabrication of PDMS-carbon electrodes for silicone dielectric elastomer transducers," in 2015 Transducers - 2015 18th International Conference on Solid-State Sensors, Actuators and Microsystems (TRANSDUCERS, pp. 1905-1908, Anchorage, AK, USA, 2015.

[67] A. O'Hallorana, F. O'Malley, and P. McHugh, “A review on dielectric elastomer actuators, technology, applications, and challenges," Journal of Applied Physics, vol. 104, no. 7, 2008.

[68] V. Nguyen, C. Kaulen, U. Simon, and U. Schnakenberg, "Single interdigital transducer approach for gravimetrical SAW sensor applications in liquid environments," Sensors, vol. 17, no. 12, p. 2931, 2017.

[69] J. Jin, S. T. Quek, and Q. Wang, "Design of interdigital transducers for crack detection in plates," Ultrasonics, vol. 43, no. 6, pp. 481-493, 2005.

[70] R. S. C. Monkhouse, P. W. Wilcox, M. J. S. Lowe, R. P. Dalton, and P. Cawley, "The rapid monitoring of structures using interdigital Lamb wave transducers," Smart Materials and Structures, vol. 9, no. 3, pp. 304-309, 2000.

[71] G. Hayward, B. Hailu, R. Farlow, A. Gachagan, and A. McNab, Design of Embedded Transducers for Structural Health Monitoring Applications, Smart Structures and Materials: Smart Structures \& Integrated Systems. International Society for Optics and Photonics, 2001.

[72] W. Ziping, Q. Lei, J. Zhengxuan, X. Xian, and R. Karthik, "Research on the optimal design and application of wideband TIDT structures," Rare Metal Materials and Engineering, vol. 49, no. 11, pp. 3790-3795, 2020.

[73] L. Li, X. Yang, Y. Yin et al., "An interdigital electrode probe for detection, localization and evaluation of surface notch-type damage in metals," Sensors, vol. 18, no. 2, p. 371, 2018.

[74] M. Mańka, M. Rosiek, A. Martowicz, T. Uhl, and T. Stępiński, "Design and simulations of interdigital transducers for Lambwave based SHM systems," 2011, https://www.researchgate .net/publication/266035698.

[75] W. Ziping, L. Ying, Z. Guoqi, and Y. Fuh-Gwo, "Design and optimization of an OPFC ultrasonic linear phased array transducer," International Journal of Mechanics and Materials in Design, vol. 13, no. 1, pp. 57-69, 2017.

[76] W. Ziping, X. Xian, L. Xingjia, and J. Zhengxuan, "Study on attenuation properties of surface wave of AE simulation source based on OPCM sensor element," Journal of Sensors, vol. 2012, 6 pages, 2018.

[77] M. Mańka, M. Rosiek, A. Martowicz, T. Stepinski, and T. Uhl, "PZT based tunable Interdigital Transducer for Lamb waves based NDT and SHM," Mechanical Systems \& Signal Processing, vol. 78, pp. 71-83, 2016.

[78] W. Peng, Y. He, C. Wen, and K. Ma, "Surface acoustic wave ultraviolet detector based on zinc oxide nanowire sensing layer," Sensors \& Actuators A Physical, vol. 184, no. 3, pp. 34-40, 2012.

[79] T. Stepinski, M. Mańka, A. Martowicz, and V. T. Rathod, "Interdigital transducers in structural health monitoring based on Lamb waves: a state of the art," in Sensors and Smart Structures Technologies for Civil, Mechanical, and Aerospace Systems 2016, Las Vegas, Nevada, USA, 2016.

[80] K. I. Salas and C. E. S. Cesnik, "Guided wave excitation by a CLoVER transducer for structural health monitoring: theory and experiments," Smart Materials and Structures, vol. 18, no. 7, article $075005,2009$.

[81] C. M. Lin, Y. Y. Chen, and T. T. Wu, "A novel weighted method for layered SAW filters using slanted finger interdigital transducers," Journal of Physics D Applied Physics, vol. 39, no. 3, pp. 466-470, 2006. 